\title{
Recent upgrades of the multiple-reflection time-of-flight mass spectrometer at TITAN, TRIUMF
}

Timo Dickel ${ }^{1,2}$ (D) . Samuel Ayet San Andrés ${ }^{1,2} \cdot$ Sönke Beck ${ }^{1,2} \cdot$ Julian Bergmann ${ }^{1}$. Jens Dilling ${ }^{3}$. Florian Greiner ${ }^{1}$. Christine Hornung ${ }^{1} \cdot$ Andrew Jacobs $^{3}$. Gabriella Kripko-Koncz ${ }^{1}$. Ania Kwiatkowski ${ }^{3}$. Erich Leistenschneider ${ }^{3}$. Alexander Pikthtelev ${ }^{4}$. Wolfgang R. Plaß ${ }^{1,2}$. Moritz P. Reiter ${ }^{1,3}$. Christoph Scheidenberger ${ }^{1,2} \cdot$ Christian Will $^{1}$ for the TITAN collaboration

Published online: 12 June 2019

(C) The Author(s) 2019

\begin{abstract}
A multiple-reflection time-of-flight mass spectrometer (MR-TOF-MS) has been installed and integrated into the TITAN experiment at TRIUMF. The MR-TOF-MS extends the capabilities and improves the performance of the TITAN experiment, for instance it provides fast isobar separation, short measurement cycles, yield measurements and broad-band beam diagnosis, and enhanced rate capabilities and dynamic range. Thereby, even more exotic nuclides become accessible at TITAN for experiments. Such improved capabilities of TITAN have been used in first experiments to investigate the sub-shell closure of neutronrich nuclides at $\mathrm{N}=32$ and the r-process nucleo-synthesis for masses at $\mathrm{A} \sim 85$. The vacuum system, electronics and data-acquisition hardware and software have been recently upgraded. For the first time an isomeric state has been measured with TITAN's MR-TOFMS, ${ }^{84 m} \mathrm{Rb}$ (excitation energy: $464 \mathrm{keV}$ ). The accurate measurement of excitation energies of low-lying isomeric states is possible due to the high mass resolving power of TITAN's MR-TOF-MS and a specialized data-analysis procedure.
\end{abstract}

Keywords Mass measurements · MR-TOF-MS · Exotic nuclides · Isomers · Instrument control · On- and offline data-analysis

This article is part of the Topical Collection on Proceedings of the 7th International Conference on Trapped Charged Particles and Fundamental Physics (TCP 2018), Traverse City, Michigan, USA, 30 September-5 October 2018

Edited by Ryan Ringle, Stefan Schwarz, Alain Lapierre, Oscar Naviliat-Cuncic, Jaideep Singh and Georg Bollen

Timo Dickel

timo.dickel@exp2.physik.uni-giessen.de

1 II. Physikalisches Institut, Justus Liebig Universität Gießen, 35392 Gießen, Germany

2 GSI Helmholtzzentrum für Schwerionenforschung GmbH, 64291 Darmstadt, Germany

3 TRIUMF, Vancouver, BC V6T 2A3, Canada

4 Institute for Energy Problems of Chemical Physics, RAS, 142432 Chernogolovka, Moscow, Russia 


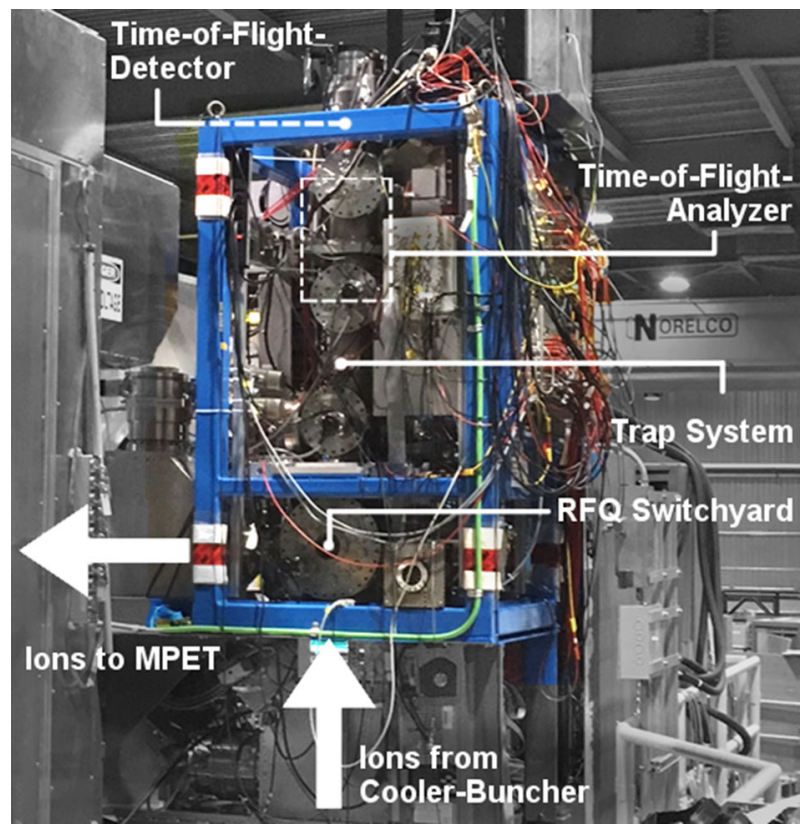

Fig. 1 Photograph of the MR-TOF-MS installed on the TITAN beamline

\section{Introduction}

TRIUMF's Ion Trap for Atomic and Nuclear science (TITAN) [1, 2] is a multiple ion-trap system capable of performing high-precision mass measurements and in-trap decay spectroscopy [3]. In particular, TITAN has specialized in fast mass spectrometry of short-lived exotic nuclei using its Penning trap [4]. In order to reach the highest possible precision and shorter measurement times, ions can be charge-bred into higher charge states by an Electron Beam Ion Trap (EBIT) [5]. Using highly-charged ions, TITAN is capable of performing mass measurements of short-lived heavy species with very high precision and accuracy [6].

Although the Isotope Separator and Accelerator (ISAC) [7] facility at TRIUMF can deliver high yields even for some of the most exotic species, some measurements suffer from a strong isobaric contamination. This contamination often prevents precision measurements of the exotic species of interest. To overcome this limitation an isobar separator and mass spectrometer based on the Multiple-Reflection Time-Of-Flight Mass Spectrometry (MR-TOF-MS) technique has been developed at the Justus Liebig University Giessen [8] and installed recently at TITAN (Fig. 1).

Ions coming from TITAN's cooler and buncher are captured in an RF quadrupole and transmitted through a novel buffer gas-filled radio-frequency quadrople (RFQ)-based switchyard [9] to the MR-TOF-MS. The MR-TOF-MS is based on the design of the MRTOF-MS developed for the MATS experiment at FAIR $[10,11]$ which is currently in use at the FRS Ion Catcher [12] at GSI. At variance to the GSI system, mass selection is achieved in the TITAN's MR-TOF-MS using dynamic re-trapping of the ions of interest after a time-of-flight analysis in an electrostatic isochronous reflector system [13, 14]. The isobarically clean ion population is transported back to the RFQ switchyard and ejected to 


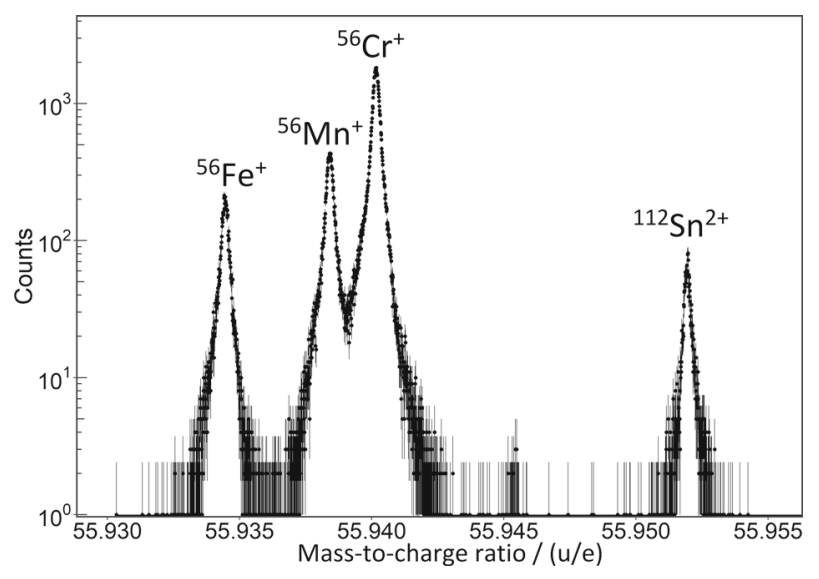

Fig. 2 Mass-to-charge spectrum near 56 u/e taken during the first online experiment with the MR-TOF-MS at TITAN. Ions were produced by a $40 \mu \mathrm{A}$ proton beam with $480 \mathrm{MeV}$ impinging on a low power Ta target coupled to a Re surface ion source. A two-step resonance-laser ionization scheme for Ti was used. The ions underwent 512 isochronous turns in the analyzer. The mass resolving power is 220,000 (FWHM)

the TITAN EBIT or Penning trap. The use of these novel techniques (RFQ-based switchyard and re-trapping) allowed the integration of the very compact MR-TOF-MS instrument with minimal impact on the existing TITAN beam line. It also provides cooled and clean isobaric beams delivered to downstream ion traps and other measurement devices. Additionally, the MR-TOF-MS enables mass measurements in itself, in particular for very short-lived nuclides that are produced at low rates, thus complementing and even extending TITAN's mass measurement program of short-lived exotic nuclei.

After a first offline [8] and online [15] commissioning phase with stable beams from ISAC in early 2017 the MR-TOF-MS was employed in a measurement campaign investigating the evolution of the $\mathrm{N}=32$ neutron-shell closure around ${ }^{52} \mathrm{Ca}$ [16]. This shell closure forms several neutrons away from stability and has been established in neutron-rich $\mathrm{K}, \mathrm{Ca}$ and Sc isotopes, whereas in the higher-Z elements, $\mathrm{V}$ and $\mathrm{Cr}$, no such shell effects had been found. High-precision mass measurements with the new MR-TOF-MS were able to prove the existence of a weak shell closure in Ti and show no shell closure in V [17]. Thereby, four of the most successful modern ab-initio theories, which all over-predict the strength of the weak $\mathrm{N}=32$ shell closure, were challenged [16].

\section{Online commissioning}

Following installation of the MR-TOF-MS on the TITAN platform, a first online run including the MR-TOF-MS was done [16, 17]. In Fig. 2 the mass-to-charge spectrum at $56 \mathrm{u} / \mathrm{e}$ measured during this experiment can be seen. The spectrum demonstrates the simultaneous measurement and identification of ions with $\mathrm{A} / \mathrm{q}=56$ with a large dynamic range of more than 3 orders of magnitude and very little background. The high mass resolving power of about 200,000 (FWHM) allows to resolve even the isobars close to stability, which have a mass excess difference of less than $2 \mathrm{MeV}$. Thereby, these isotopes with well known masses can be used for the calibration of the mass spectrum. Doubly-charged ions, like in this case ${ }^{112} \mathrm{Sn}$, can also be measured and identified. A more detailed description of the performance of the system will be subject of a separate, forthcoming publication [15]. 


\section{Upgrades of electronics, vacuum and data-acquisition hardware}

The high-voltage (HV) switches, RC low-pass filters and power supplies for the voltages of the analyzer were reworked to improve the stability and reliability. The new electronics results in reduced electric ringing and more precise voltages on the analyzer electrodes.

The transmission efficiency of the analyzer has been improved by a factor 2 for high number ( $\sim 400)$ of isochronous turns, i.e. for high mass resolving powers. This was achieved by installing an additional turbo-molecular pump on the analyzer section (about doubling the pumping speed on this vacuum section), which effectively reduces the residual gas pressure in the time-of-flight analyzer.

The previously installed MCP detector [8] was replaced with a MagneTOF detector. The new detector has about a factor 2 higher detection efficiency. In addition, it offers better signal quality, i.e. less ringing and more than an order of magnitude lower dark-count rate, and a position independent detection efficiency.

The data-acquisition system supports time-to-digital converter (TDC) and analog-todigital converters (ADC). Recently, the Ortec-9353 TDC has been replaced by a FASTComTec MCS6A. The new TDC has zero deadtime and thereby about a factor 2 higher event rates can now be handled by the system.

Together, these measures increase the total efficiency by about a factor $\sim 4$, the system is more stable and reliable, and longer measurement times are enabled $(\sim 10 \mathrm{~h}$ instead of few hours). Thereby, the MR-TOF-MS reaches at least one mass unit more exotic nuclides than before.

\section{New software for instrument control, data acquisition and data analysis}

For an efficient and reliable operation of a high-performance system, such as this MR-TOFMS, a high-level control system for voltages, timings etc. is essential. All MR-TOF-MS built at the Justus Liebig University Giessen $[8,11,18]$ use the same custom developed softwares for experiment control, data acquisition, storage and analysis.

The system control software allows to control and display hundreds of voltage channels, but also has many useful features for reliable and easy online operation: grouping of channels, setting voltages and currents by formulas (cross referencing of channels), connecting channels, data logging, remote access via TCP-server, just to name a few.

The timing control is integrated in the data-acquisition and on-line data-analysis software (Mass Acquisition, MAc) [19]. This software has been developed and is maintained by the group from Justus Liebig University Giessen. MAc can also control the voltages of the device via a link to the system control software. MAc offers a variety of methods of adaptive data analysis and supports multiple detection hardwares, each one offering specific advantages. The data analysis is specialized for MR-TOF-MS. The combination of these features allow MAc to create control loops and optimization procedures including automatic data analysis. Furthermore, MAc allows a broad range of data-visualization modes and export features to integrate seamlessly into other, already existing workflows. The identification of peaks in complex spectra, with high turn number and broad mass range, is particularly challenging. In MR-TOF-MS the conversion of the measured TOF to a mass-to-charge ratio is ambiguous and each mass-to-charge ratio can, within its uncertainty, correspond to many different combinations of isotopes (including molecules, adducts, etc.). A peak 

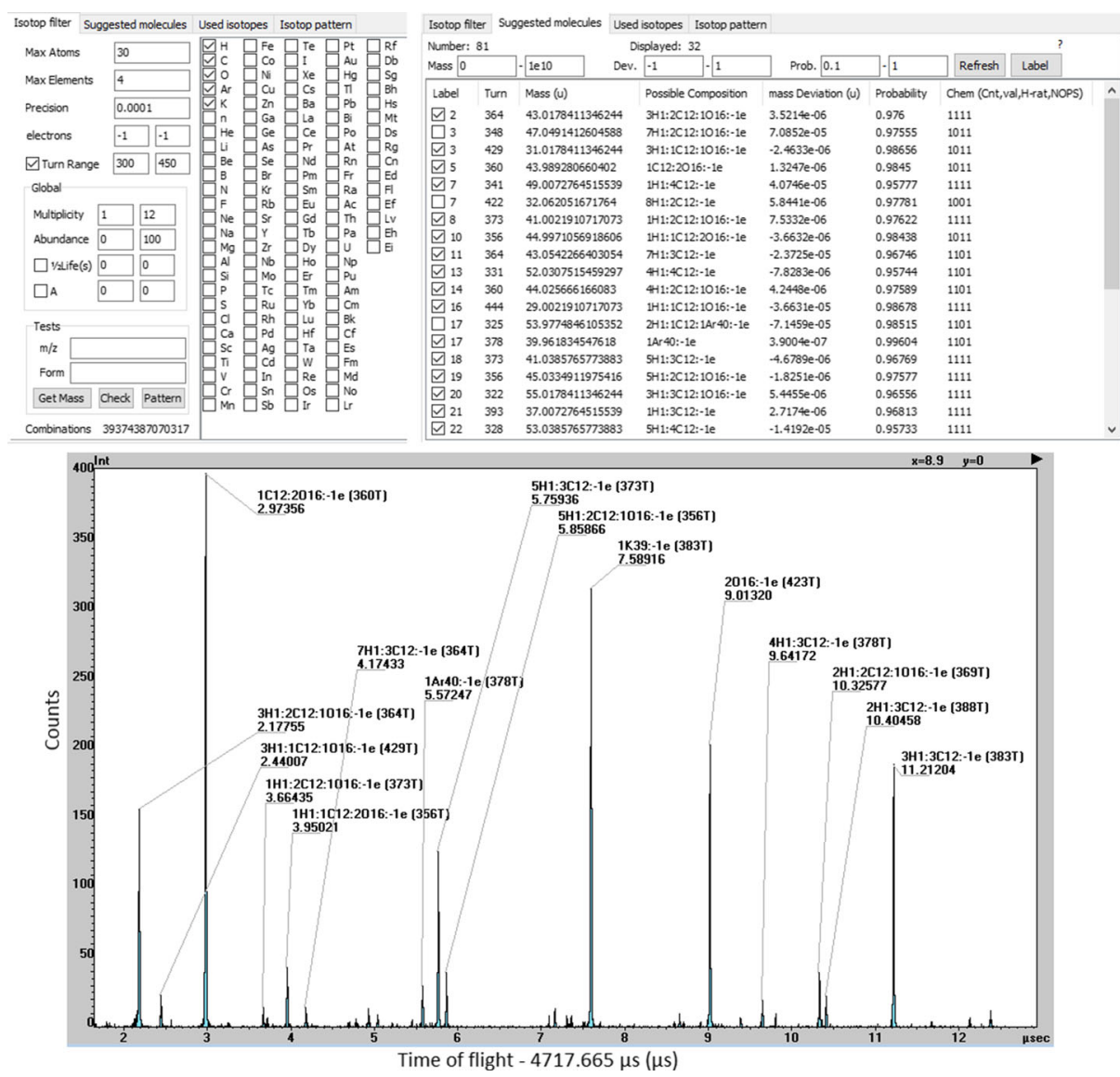

Fig. 3 Example for the automated peak identification in MAc. In the top left the menu for input parameters (mass, element and turn-number range, etc.) is displayed. The top right shows the output with the list of possible hits for the peaks seen in the mass spectrum below. The bottom shows the measured spectrum with labels for the identified peaks. The present spectrum was obtained merging natural $\mathrm{K}$ ions from a thermal ion source (Heatwave Labs) with ions created from impurities of the He buffer gas, ionized by the filament of a hot-cathode vacuum ion gauge positioned next to the internal RFQ beam transport system of the TITAN's MR-TOF-MS. All ions were transported to the injection RF trap, where they were cooled for $10 \mathrm{~ms}$ prior to the mass measurement. The electronic switches of the time-of-flight mass analyzer were adjusted such that ${ }^{39} \mathrm{~K}^{+}$ions underwent 383 turns and ions with mass from $32 \mathrm{u}$ to $55 \mathrm{u}$ underwent 460 to 322 turns, respectively

identification routine was developed to cope with this problem. It allows for selections on the elements, accuracy, half-life, abundance and for the possible combinations of isotopes the mass deviation, basic rules of molecular chemistry are checked and the product of the abundances can be used to select the correct identification. The guidelines for chemical correctness are based on molecular stability and the heuristic occurrence of the elements within certain molecules [20]. In order to obtain a correct identification, an iterative procedure is applied, where four rules are evaluated, which are in the present case:

- Maximum occurrences of C, H, N, O, P, S, F, Cl, Br, Si in molecules below mass 2000 u.

- $\quad$ Rules (LEWIS+ and SENIOR) based on the number of valence electrons. 


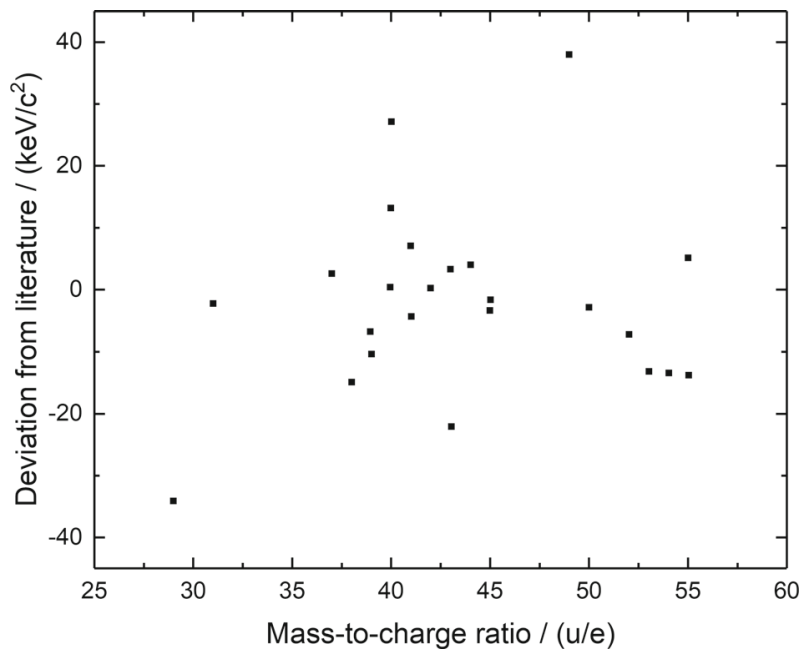

Fig. 4 Deviation of mass as determined in the online analysis with MAc and the literature mass of the identified ions for Fig. 3. The standard deviation is $15 \mathrm{keV} / \mathrm{c}^{2}$, showing the accuracy of the online analysis

- For the same elements the ratio to $\mathrm{H}$ needs to be in a certain range.

- Occurrence ranges of N, O, P, S in molecules composed of at least three of these elements (molecule mass below $2000 \mathrm{u}$ ).

The peak identification with its intermediate steps and the final result is shown for an example in Fig. 3. The complete process takes about $15 \mathrm{~min}$, from the end of the measurement to the identification as shown in Fig. 3. There, 27 out of 38 peaks (that are higher than 1 event per bin) were identified. This online mass calibration and peak identification already provides a highly accurate mass determination, see Fig. 4. In the example presented here, the average deviation from the literature masses of the identified ions is $(-2 \pm 3) \mathrm{keV} / \mathrm{c}^{2}$ for on average of $\sim 400$ detected ions per species.

\section{Low-lying isomeric states}

Measuring and resolving low-lying isomeric states has always been a challenge for mass spectrometers and is met only by the ones, which reach highest resolving powers. The MRTOF-MS of the TITAN experiment has achieved high mass resolving powers above 200,000 and is thereby able to detect isomers with excitation energies of more than $500 \mathrm{keV}$ for medium-heavy nuclides. The first isomeric state measured with the MR-TOF-MS at TITAN is ${ }^{84 m} \mathrm{Rb}$, see Fig. 5.

To measure the excitation energy accurately, the peak shape needs to be known very precisely and the data-analysis procedure needs to be able to cope with these special challenges. Such a procedure has been developed for the MR-TOF-MS at GSI [21] and is now applied for measurements with TITAN's MR-TOF-MS. As an example, the measurement of ${ }^{84} \mathrm{Rb}$ in its ground and isomeric state is shown in Fig. $5 .{ }^{84 m} \mathrm{Rb}$ was also the first isomeric state resolved by mass spectrometry more than 25 years ago [22]. Even though, in Fig. 5, there are multiple mass distributions partially overlapping, the measured excitation energy of (474 \pm 12$) \mathrm{keV}$ agrees with the literature value of (463.59 \pm 0.08$) \mathrm{keV}$ [23]. 


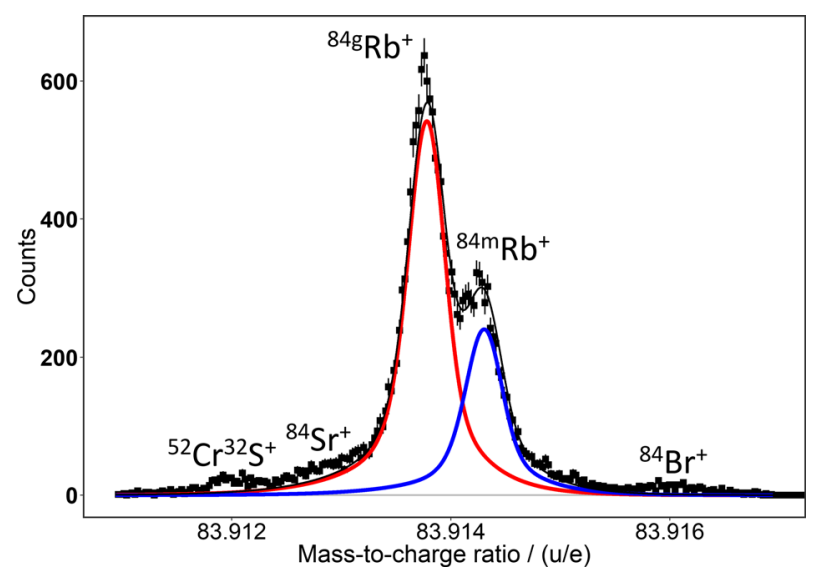

Fig. 5 About $16000{ }^{84} \mathrm{Rb}$ ions were measured, $\sim 30 \%$ of which were produced in isomeric state. The measured excitation energy of the isomeric state of ${ }^{84} \mathrm{Rb}$ is $(474 \pm 12) \mathrm{keV}$. The ions were stored in the time-of-flight analyzer for 332 isochronous turns, corresponding to a total time-of-flight of $\sim 6.005 \mathrm{~ms}$

\section{Summary and Outlook}

Being able to detect and separate isobars and isomers and at the same time measure a broadband mass range, the new MR-TOF-MS has become a standard device in recent experiments at TITAN. Upgrades to the electronics, detector and vacuum system enable up to a factor 4 higher detection efficiency and higher mass accuracy. The dedicated and specially developed instrument-control and data-acquisition and analysis softwares enables the real-time determination of the radioactive-beam composition and optimization of the ISAC mass separator, for precision mass measurements and, in the near future, for isobar separation. The device can be used as isobar separator and in the same measurement cycle for direct mass measurements. Accurate measurements of excitation energies of isomers, as shown for the case of ${ }^{84 m} \mathrm{Rb}$, are possible due to the high resolving power $(>200,000)$ and the data analysis adopted from the MR-TOF-MS at the FRS at GSI in Darmstadt, Germany.

Acknowledgements We want to thanks Ryan Ringle for valuable input and discussion in the development of the peak identification routines. We thank Christian Lotze for his work on the voltage control software. This work was supported by National Science and Engineering Research Council of Canada (NSERC), federal funding through TRIUMF by the National Research Council of Canada (NRC), by the German Federal Ministry for Education and Research (BMBF) under under contracts no. 05P19RGFN1, 05P12RGFN8 and 05P15RGFN1, by Justus-Liebig-Universität Gießen and GSI under the JLU-GSI strategic Helmholtzpartnership agreement, by HGS-HIRe, and by the Hessian Ministry for Science and Art (HMWK) through the LOEWE Center HICforFAIR and the LOEWE Focus AmbiProbe.

Open Access This article is distributed under the terms of the Creative Commons Attribution 4.0 International License (http://creativecommons.org/licenses/by/4.0/), which permits unrestricted use, distribution, and reproduction in any medium, provided you give appropriate credit to the original author(s) and the source, provide a link to the Creative Commons license, and indicate if changes were made. 


\section{References}

1. Dilling, J., Baartman, R., Bricault, P., Brodeur, M., Blomeley, L., Buchinger, F., Crawford, J., LópezUrrutia, J.C., Delheij, P., Froese, M., Gwinner, G., Ke, Z., Lee, J., Moore, R., Ryjkov, V., Sikler, G., Smith, M., Ullrich, J., Vaz, J.: Int. J. Mass Spectr. 251(2), 198 (2006)

2. Kwiatkowski, A.A., Andreoiu, C., Bale, J.C., Brunner, T., Chaudhuri, A., Chowdhury, U., Delheij, P., Ettenauer, S., Frekers, D., Gallant, A.T., Grossheim, A., Gwinner, G., Jang, F., Lennarz, A., Ma, T., Mané, E., Pearson, M.R., Schultz, B.E.M.: Hyperfine Interact. 225(1), 143 (2014)

3. Lennarz, A., Grossheim, A., Leach, K.G., Alanssari, M., Brunner, T., Chaudhuri, A., Chowdhury, U., Crespo López-Urrutia, J.R., Gallant, A.T., Holl, M., Kwiatkowski, A.A., Lassen, J., Macdonald, T.D., Schultz, B.E., Seeraji, S., Simon, M.C., Andreoiu, C., Dilling, J., Frekers, D.: Phys. Rev. Lett. 113, 082502 (2014). https://doi.org/10.1103/PhysRevLett.113.082502. https://link.aps.org/doi/10.1103/ PhysRevLett.113.082502

4. Brodeur, M., Brunner, T., Champagne, C., Ettenauer, S., Smith, M., Lapierre, A., Ringle, R., Ryjkov, V.L., Audi, G., Delheij, P., Lunney, D., Dilling, J.: Phys. Rev. C 80, 044318 (2009). https://doi.org/10.1103/PhysRevC.80.044318. https://link.aps.org/doi/10.1103/PhysRevC.80.044318

5. Lapierre, A., Brodeur, M., Brunner, T., Ettenauer, S., Gallant, A., Simon, V., Good, M., Froese, M., López-Urrutia, J.C., Delheij, P., Epp, S., Ringle, R., Schwarz, S., Ullrich, J., Dilling, J.: Nucl. Inst. Methods Phys. Res. A: Accelerators, Spectrometers, Detectors and Associated Equipment 624(1), 54 (2010)

6. Malbrunot-Ettenauer, S., Brunner, T., Chowdhury, U., Gallant, A.T., Simon, V.V., Brodeur, M., Chaudhuri, A., Mané, E., Simon, M.C., Andreoiu, C., Audi, G., Crespo López-Urrutia, J.R., Delheij, P., Gwinner, G., Lapierre, A., Lunney, D., Pearson, M.R., Ringle, R., Ullrich, J., Dilling, J.: Phys. Rev. C 91, 045504 (2015). https://doi.org/10.1103/PhysRevC.91.045504. https://link.aps.org/doi/10.1103/ PhysRevC.91.045504

7. Dombsky, M., Bishop, D., Bricault, P., Dale, D., Hurst, A., Jayamanna, K., Keitel, R., Olivo, M., Schmor, P., Stanford, G.: Rev. Sci. Instrum. 71, 978 (2000)

8. Jesch, C., Dickel, T., Plaß, W.R., Short, D., Ayet San Andrés, S., Dilling, J., Geissel, H., Greiner, F., Lang, J., Leach, K.G., Lippert, W., Scheidenberger, C., Yavor, M.I.: Hyperfine Interact. 235(1), 97 (2015)

9. Plaß, W.R., Dickel, T., Ayet San Andrés, S., Ebert, J., Greiner, F., Hornung, C., Jesch, C., Lang, J., Lippert, W., Majoros, T., Short, D., Geissel, H., Haettner, E., Reiter, M.P., Rink, A.K., Scheidenberger, C., Yavor, M.I.: Phys. Scr. T166, 014069 (2015)

10. Plaß, W.R., Dickel, T., Czok, U., Geissel, H., Petrick, M., Reinheimer, K., Scheidenberger, C., Yavor, M.: Nucl. Instrum. Methods B 266(19-20), 4560 (2008)

11. Dickel, T., Plaß, W.R., Becker, A., Czok, U., Geissel, H., Haettner, E., Jesch, C., Kinsel, W., Petrick, M., Scheidenberger, C., Simon, A., Yavor, M.I.: Nucl. Instrum. Methods A 777, 172 (2015)

12. Plaß, W.R., Dickel, T., Scheidenberger, C.: Int. J. Mass Spectrom. 349-350, 134 (2013)

13. Dickel, T., Yavor, M.I., Lang, J., Plaß, W.R., Lippert, W., Geissel, H., Scheidenberger, C.: Int. J. Mass Spectrom. 412, 1 (2017)

14. Dickel, T., Plaß, W.R., Lippert, W., Lang, J., Yavor, M.I., Geissel, H., Scheidenberger, C.: J. Am. Soc. Mass Spectr. 28(6), 1079 (2017)

15. Reiter, M.P., et al.: Manuscript pending submission (2019)

16. Leistenschneider, E., Reiter, M.P., Ayet San Andrés, S., Kootte, B., Holt, J.D., Navrátil, P., Babcock, C., Barbieri, C., Barquest, B.R., Bergmann, J., Bollig, J., Brunner, T., Dunling, E., Finlay, A., Geissel, H., Graham, L., Greiner, F., Hergert, H., Hornung, C., Jesch, C., Klawitter, R., Lan, Y., Lascar, D., Leach, K.G., Lippert, W., McKay, J.E., Paul, S.F., Schwenk, A., Short, D., Simonis, J., Somà, V., Steinbrügge, R., Stroberg, S.R., Thompson, R., Wieser, M.E., Will, C., Yavor, M., Andreoiu, C., Dickel, T., Dillmann, I., Gwinner, G., Plaß, W.R., Scheidenberger, C., Kwiatkowski, A.A., Dilling, J.: Phys. Rev. Lett. 120, 062503 (2018)

17. Reiter, M.P., Ayet San Andrés, S., Dunling, E., Kootte, B., Leistenschneider, E., Andreoiu, C., Babcock, C., Barquest, B.R., Bollig, J., Brunner, T., Dillmann, I., Finlay, A., Gwinner, G., Graham, L., Holt, J.D., Hornung, C., Jesch, C., Klawitter, R., Lan, Y., Lascar, D., McKay, J.E., Paul, S.F., Steinbrügge, R., Thompson, R., Tracy, J.L., Wieser, M.E., Will, C., Dickel, T., Plaß, W.R., Scheidenberger, C., Kwiatkowski, A.A., Dilling, J.: Phys. Rev. C 98, 024310 (2018)

18. Dickel, T., Plaß, W., Lang, J., Ebert, J., Geissel, H., Haettner, E., Jesch, C., Lippert, W., Petrick, M., Scheidenberger, C., Yavor, M.: Nucl. Inst. Methods Phys. Res. B: Beam Interactions with Materials and Atoms 317, 779 (2013). XVIth International Conference on ElectroMagnetic Isotope Separators and Techniques Related to their Applications, December 2-7, 2012 at Matsue, Japan 
19. Bergmann, J.: Datenaufnahme und Systemsteuerung eines Flugzeitmassenspektrometers mit Anwendungen. Master thesis, Universität Gießen (2015)

20. Kind, T., Fiehn, O.: BMC Bioinf. 8, 105 (2007)

21. Ayet San Andrés, S., et al.: Submitted to PRC, arXiv:1901.11278 (2019)

22. Bollen, G., Kluge, H.J., König, M., Otto, T., Savard, G., Stolzenberg, H., Moore, R., Rouleau, G., Audi, G.: Phys. Rev. C 46(6), R2140 (1992)

23. Audi, G., Kondev, F.G., Wang, M., Huang, W.J., Naimi, S.: Chin. Phys. C 41(3), 030001 (2017)

Publisher's note Springer Nature remains neutral with regard to jurisdictional claims in published maps and institutional affiliations. 